\title{
Tuning phase transitions of FeSe thin flakes by field effect transistor with solid ion conductor as gate dielectric
}

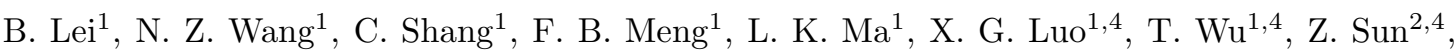

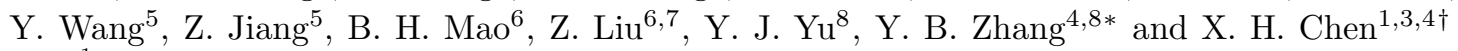 \\ ${ }^{1}$ Hefei National Laboratory for Physical Sciences at Microscale and Department of Physics, \\ and CAS Key Laboratory of Strongly-coupled Quantum Matter Physics, \\ University of Science and Technology of China, Hefei, Anhui 230026, China \\ ${ }^{2}$ National Synchrotron Radiation Laboratory, University of Science and Technology of China, Hefei, Anhui 230026, China \\ ${ }^{3}$ High Magnetic Field Laboratory, Chinese Academy of Sciences, Hefei, Anhui 230031, China \\ ${ }^{4}$ Collaborative Innovation Center of Advanced Microstructures, Nanjing University, Nanjing 210093, China \\ ${ }^{5}$ Shanghai Institute of Applied Physics, Chinese Academy of Sciences, Shanghai 201204, China \\ ${ }^{6}$ State Key Laboratory of Functional Materials for Informatics, Shanghai Institute of Microsystem \\ and Information Technology, Chinese Academy of Sciences, Shanghai 200050, China \\ ${ }^{7}$ School of Physical Science and Technology, Shanghai Tech University, Shanghai 200031, China \\ ${ }^{8}$ State Key Laboratory of Surface Physics and Department of Physics, Fudan University, Shanghai 200433, China
}

(Dated: October 18, 2018)

\begin{abstract}
We develop a novel field effect transistor (FET) device using solid ion conductor (SIC) as a gate dielectric, and we can tune the carrier density of FeSe by driving lithium ions in and out of the FeSe thin flakes, and consequently control the material properties and its phase transitions. A dome-shaped superconducting phase diagram was mapped out with increasing Li content, with $T_{c}$ $\sim 46.6 \mathrm{~K}$ for the optimal doping, and an insulating phase was reached at the extremely overdoped regime. Our study suggests that, using solid ion conductor as a gate dielectric, the SIC-FET device can achieve much higher carrier doping in the bulk, and suit many surface sensitive experimental probes, and can stabilize novel structural phases that are inaccessible in ordinary conditions.
\end{abstract}

PACS numbers: 74.25.F-, 74.70.Xa, 74.78.-w

Chemical doping is a conventional way to introduce charge carriers into solids by replacing one of the constituent elements with another element of a different valence state. For instance, high temperature superconductivity is realized by suppressing the antiferromagnetism or spin density wave with chemical doping of $10 \%$ or $10^{21}$ dopant atoms per $\mathrm{cm}^{3}$ in copper oxides and iron-based superconductors [1-3]. However, the chemical doping is incapable in many cases, because the element replacement and the variation of carrier density cannot practically cover a large regime and leave many phases unexplored. As a complementary method, the application of field effect transistors (FET) in two-dimensional systems is an effective way to control electronic properties via reversible changes of charge carrier density [4 18]. Such an electrostatic doping is desirable to study novel phases that cannot be achieved by material synthetic methods [7, 9 11, 13, 15, 16, 18]. For instance, we have utilized tunable ion intercalation with an ionic liquid to alter charge-ordered states in $1 \mathrm{~T}-\mathrm{TaS}_{2}$ and induce phase transitions in thin flakes with reduced dimensionality [15]. The FET devices have been widely applied in the exploration of new superconductors [10, 11], the preparation for new devices [19, 20] as well as many applications in semiconductor industry [7].

So far, only two types of field effect transistor (FET) devices, metal-insulator-semiconductor (MIS) FET (Fig. 1(a)) and electric double layer (EDL) FET (Fig. 1(b)), can be widely used to continuously tune carrier density
21, 22]. However, there are inherent drawbacks for the investigation of novel quantum phases in solids. In conventional MIS-FET devices, the electrostatic doping to a system is realized by the accumulation of mobile carriers at the surface of an insulator with a gate voltage applied 6, 7, 11, 21, 22]. A limited sheet carrier density $\mathrm{n}_{2 D}$ of $\sim 1 \times 10^{13} \mathrm{~cm}^{-2}$ can be obtained on the surface of an insulator before the gate dielectric breaks down due to the large electric field [5, 9, 11]. They cannot provide sufficient carriers to induce superconductivity [22]. The EDL-FET with a liquid electrolyte as a gate dielectric can achieve a higher two-dimensional carrier density $\mathrm{n}_{2 D}$ of $\sim 8 \times 10^{14} \mathrm{~cm}^{-2}$ 23]. However, the overlay of liquid electrolyte makes it difficult to suit the modern electronic technology and prevents heavily-doped electronic states from being characterized by many physical measurements. In addition, many materials of interest have electrochemical reaction with liquid electrolyte [24, 25]. Moreover, both of FETs can only tune the accumulation of carrier on the surface of materials. In this letter, using solid ion conductor as a gate dielectric, we introduce a new type of FET, SIC-FET (Fig. 1(c)). This type of FET devices solves the shortcoming of the carrier control methods mentioned above and can pave the way for the investigation of new electronic states in solids.

To demonstrate the capability of the SIC-FET device, we choose FeSe as a model material. FeSe has the simplest structure in iron-based high-temperature superconductors, with conducting FeSe layers stacked along c-axis 
(a)

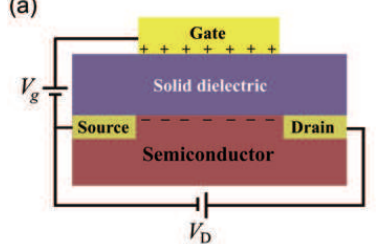

(c)

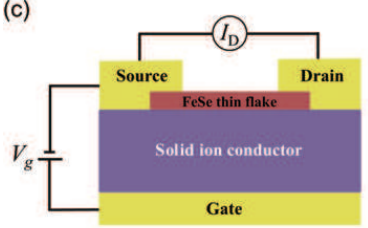

(e)

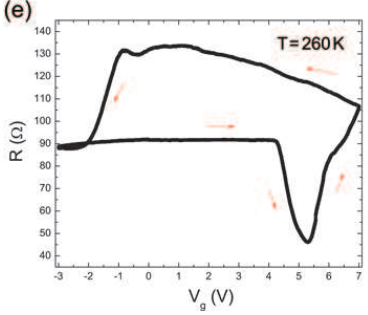

(b)

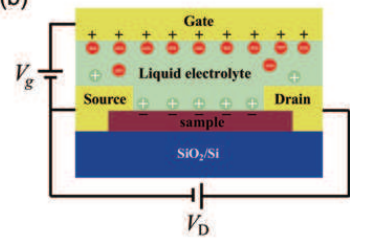

(d)
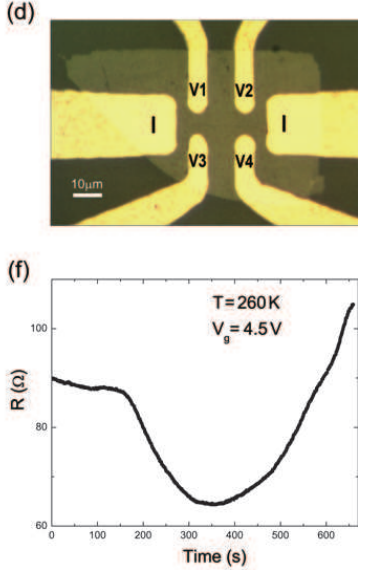

FIG. 1: (color online). Resistance controlled by gate voltage with SIC-FET device. (a) A schematic illustration of the MIS-FET with solid oxide such as $\mathrm{SiO}_{2}$ or $\mathrm{SrTiO}_{3}$ as the gate dielectric; (b) A schematic structure of EDL-FET with liquid electrolyte as the gate dielectric; (c) A schematic diagram of the SIC-FET device with the solid ion conductor as the gate dielectric; (d) The optical image of a FeSe thin flake with a standard Hall bar configuration with the current and voltage terminals labeled; (e) Gate voltage dependence of the resistance for a FeSe thin flake with typical thickness of about 18 $\mathrm{nm}$ in SIC-FET device. The continuously swept gate voltage with a scan rate of $2 \mathrm{mVs}^{-1}$ was applied at $260 \mathrm{~K}$; (f) The evolution of the resistance with time in SIC-FET with $\mathrm{V}_{g}=$ $4.5 \mathrm{~V}$ where the resistance of the sample started to drop.

[26]. FeSe and its derived superconductors are currently the focus of research in the field of iron-based superconductors [27 31]. In particular, the monolayer FeSe thin film on $\mathrm{SrTiO}_{3}$ substrate has generated wide research interest because of its unexpected high- $T_{c}$ superconductivity with $T_{c}$ as high as $65 \mathrm{~K}$, in sharp contrast to the bulk $T_{c}<10 \mathrm{~K}$ [27, 31 36]. Numerous studies have shown that those FeSe-derived materials with $T_{c}>40 \mathrm{~K}$ are heavily electron-doped systems, which share very similar electronic structures [37, 38]. The origin of superconductivity in these high- $T_{c}$ materials remains to be an intriguing topic.

Recently, we have systematically tuned the superconductivity of FeSe thin flakes by electron doping with a FET device [39]. A onset high- $T_{c}$ of $48 \mathrm{~K}$ was achieved in FeSe thin flakes with initial $T_{c}$ less than $10 \mathrm{~K}$. Intriguingly, a Lifshitz transition occurs at a certain carrier concentration, leading to a sudden change of $T_{c}$ from less than $10 \mathrm{~K}$ to more than $30 \mathrm{~K}$ [39]. However, the superconducting regime in the carrier-doping phase di-

agram is incomplete due to the sample damage caused by electrochemical reactions between samples and ionic liquid at high gate voltage $\left(V_{g}>6 \mathrm{~V}\right)$. To investigate the whole superconducting regime, a more effective method is required to introduce higher carrier density into FeSe.

Using the newly developed SIC-FET devices to increase electron doping in FeSe thin flakes by driving $\mathrm{Li}^{+}$ ions into samples, we have found structural transitions from $\mathrm{FeSe}$ (11) phase to a low-doping $\mathrm{Li}_{y} \mathrm{Fe}_{2} \mathrm{Se}_{2}$ (122 phase-I), then to a high-doping 122 phase-II. A domeshaped superconducting phase diagram was mapped out with increasing $\mathrm{Li}$ content, and $T_{c}$ is enhanced from 8 $\mathrm{K}$ in FeSe to $46.6 \mathrm{~K}$ for the optimal doping, then decreases in the overdoped regime and eventually an insulating phase emerges. The SIC-FET device proves to be able to introduce much higher carrier doping in bulk materials and stabilize novel structural phases. The application of such a novel FET device can provide exciting opportunities for exploring new quantum phases and new materials.

We prepared FET devices with the solid state lithium ion conductive glass ceramics as the gate dielectrics. The exfoliated FeSe thin flakes with a typical thickness of $\sim 18$ nm were used to fabricate the transport channel. $\mathrm{Li}^{+}$in the lithium ion conductor can move under the applied electric field. For positive gate voltage, $\mathrm{Li}^{+}$accumulates on the surface of samples, and then enters into samples to tune the carrier concentration. Figure 1(c) depicts a schematic illustration of the SIC-FET device. Detailed device preparation procedures are described in the Supplemental Material. Fig. 1(d) shows the optical image of a FeSe thin flake with a standard Hall bar configuration and with current and voltage terminals labeled. A continuously swept positive gate voltage with a scan rate of $2 \mathrm{mVs}^{-1}$ was applied at $260 \mathrm{~K}$, which is the optimal temperature for applying gate voltages. A typical $R-V_{g}$ curve is shown in Fig. 1(e). The resistance of the FeSe thin flake remains almost unchanged with $V_{g}<4.5 \mathrm{~V}$, and starts to drop quickly for $V_{g}>4.5 \mathrm{~V}$, and reaches a minimum at $5.3 \mathrm{~V}$, then increases rapidly. When the gate voltage is swept back to $-2 \mathrm{~V}$, the resistance can recover to the initial value. This behavior indicates that the tuning process is reversible. In fact, when the resistance of the sample starts to drop, the process of $\mathrm{Li}^{+}$ intercalating into the FeSe thin flake sample will continue even if the gate voltage stays at a certain level. As shown in Fig. 1(f), a continuous change of the resistance of a sample in the relaxation process at a fixed gate voltage and temperature is quite similar to that with continuous sweeping of the gate voltage.

In FeSe-derived superconductors, numerous studies suggest that carrier concentration doped into FeSe layers is the primary factor that controls the superconducting transition temperature. When $\mathrm{Li}^{+}$ions in lithium ion conductor is driven to a FeSe think flake with gating electric field, we can effectively introduce electron carri- 
ers into FeSe layers. After intercalating $\mathrm{Li}^{+}$into FeSe thin flakes at $260 \mathrm{~K}$, we quickly cooled down the FET devices to $210 \mathrm{~K}$, below which the mobility of $\mathrm{Li}^{+}$ions in thin flake samples is completely suppressed. The gate voltage of $4.5 \mathrm{~V}$, at which the resistance of sample starts to drop, was kept fixed during the whole measurements. In order to achieve a fine modulation of $\mathrm{Li}^{+}$ions intercalation, the FET device was relaxed at $260 \mathrm{~K}$ for a certain period of time before cooling down in each round. This procedure can lead to a series of doping levels with a controllable fashion.

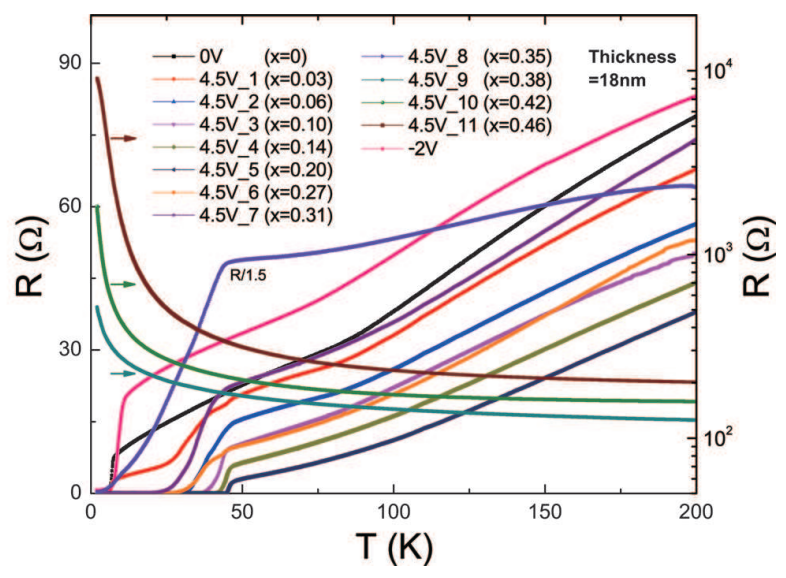

FIG. 2: (color online). Temperature dependence of resistance for a FeSe thin flake without gating and with gate voltage $\mathrm{V}_{g}=4.5 \mathrm{~V}$. The thickness of the thin flake determined by atomic force microscopy is $18 \mathrm{~nm}$. The $x$ is Li:Fe ratio whose determination is discussed in the Supplemental Material.

Using the four-terminal configuration, we measured a FeSe thin flake with various amounts of intercalated $\mathrm{Li}^{+}$ ions below $200 \mathrm{~K}$ and show them in Fig. 2. At $V_{g}=0$ $\mathrm{V}$, the sample is superconducting with the onset critical temperature $T_{c}^{\text {onset }}=7.3 \mathrm{~K}$ and reaches zero-resistance at $5 \mathrm{~K}$. Here, $T_{c}^{\text {onset }}$ is defined as the intersection between the linear extrapolation of the normal state and the superconducting transition. With increasing the amount of intercalated $\mathrm{Li}^{+}$ions, the electronic carrier density of the sample increases (as shown in Fig. S2(b) of Supplemental Material), and the $T_{c}$ as a function of $\mathrm{Li}^{+}$concentration shows a dome-like behavior. For the fifth round of cooling down at $V_{g}=4.5 \mathrm{~V}$, the optimal superconductivity of the FeSe thin flake has been obtained with the $\mathrm{Li} / \mathrm{Fe}$ ratio of 0.2 . With further increasing the amount of intercalated $\mathrm{Li}^{+}$ions, the $T_{c}$ starts to decrease, and eventually the FeSe thin flake shows an insulating behavior. When the gate voltage is swept back to $-2 \mathrm{~V}$, the resistance comes back to the initial value and the $T_{c}$ also turns back to about $8 \mathrm{~K}$, being almost the same as the initial $T_{c}$ before gating. This behavior further indicates that the carrier tuning procedure with the SIC-FET device is reversible, though the evident higher resistance indicates that the intercalation of $\mathrm{Li}^{+}$ions into the system may damage the sample in some way. We note here that both the optimal $T_{c}^{\text {onset }}=46.6 \mathrm{~K}$ and the transition from the superconductivity to insulating behavior were repeatable in every measured device. The sample with optimal doping exhibits $T_{c}^{\text {onset }}=46.6 \mathrm{~K}$ and zero-resistance temperature $T_{c}^{z e r o}$ of $44.8 \mathrm{~K}$, which is slightly higher than that of Li/ammonia intercalated FeSe synthesized by the ammonothermal method [29]. The width of the superconducting transition is less than $2 \mathrm{~K}$, much sharper than that of $\sim 13 \mathrm{~K}$ observed by tuning carrier concentration with EDL-FET device for FeSe thin flakes and thin films [39, 40] (see Fig. S3). This contrast suggests that the carrier concentration distribution in the SIC-FET devices is more homogeneous than that in the EDL-FET devices.

Owing to the small ionic radius of $\mathrm{Li}^{+}$ions, they can be easily intercalated into layered materials and alter local crystal structures of materials. Figure 3 shows the in-situ X-ray diffraction (XRD) patterns for a FeSe thin flake with thickness of $40 \mathrm{~nm}$ before and after the intercalation of $\mathrm{Li}^{+}$ions. Before the intercalation of $\mathrm{Li}^{+}$, the diffraction peak of (001) appears at $2 \theta=16.09^{\circ}$. With increasing the amount of intercalated $\mathrm{Li}^{+}$ions, the (001) peak intensity shows a drastic decrease with no noticeable variation in position. The phase with extremely low amount of $\mathrm{Li}^{+}$ions possesses the same structure as that of FeSe (11 phase), in which $\mathrm{Li}^{+}$ions diffuse randomly in the FeSe crystal. With the increase of $\mathrm{Li}^{+}$ions, two additional diffraction peaks appear at lower angles $13.76^{\circ}$ and $13.29^{\circ}$, indicating new phases derived from the structure of FeSe. These new peaks do not belong to the same phase because only the $13.29^{\circ}$ peak shows up in the insulating phase (see Fig. 3b). The data suggests that the intercalation of $\mathrm{Li}^{+}$ions into FeSe thin flakes results in two new phases.
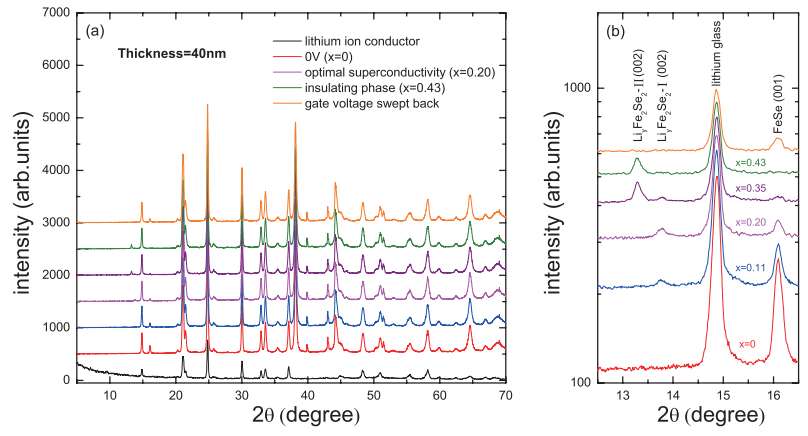

FIG. 3: (color online). In-situ X-ray diffraction (XRD) patterns for the FeSe thin flake in SIC-FET device before and after the intercalation of $\mathrm{Li}^{+}$ions. (a) The typical XRD patterns of the FeSe thin flakes with thickness of $40 \mathrm{~nm}$ for different charge stages; (b) The magnified view of the angle range from $12.5^{\circ}$ to $16.5^{\circ}$.

To identify the phases with stronger signals, we carried out XRD measurements on a thicker flake of 150 nm. As shown in Fig. S5 in the Supplemental Material, two sets of $(00 l)$ diffraction peaks have been identified, 
suggesting that the separations between adjacent Fe layers $(d)$ are 6.4128 Aand $6.6570 \AA$, respectively. In the $\mathrm{Na}_{0.65} \mathrm{Fe}_{1.93} \mathrm{Se}_{2}$ and $\mathrm{K}_{x} \mathrm{Fe}_{2-y} \mathrm{Se}_{2}$ of $\mathrm{ThCr}_{2} \mathrm{Si}_{2}$-type structure, the separations between neighboring FeSe layers are 6.8339 Åand 7.0180 A, respectively [27, 41]. Considering that the ionic radius of $\mathrm{Li}^{+}$is smaller than that of $\mathrm{Na}^{+}$and $\mathrm{K}^{+}$, we attribute the two new phases to the $\mathrm{ThCr}_{2} \mathrm{Si}_{2}$-type structure. We label the phase with a smaller lattice parameter $c(12.8256 \AA)$ as $\mathrm{Li}_{y} \mathrm{Fe}_{2} \mathrm{Se}_{2}-\mathrm{I}$ (122-I) phase and the one with larger $\mathbf{c}(13.314 \AA)$ as $\mathrm{Li}_{y} \mathrm{Fe}_{2} \mathrm{Se}_{2}$-II (122-II) phase. The different layer separation $\mathrm{d}$ in the two phases can be attributed to the different $\mathrm{Li}^{+}$contents. Generally speaking, the small cation may have more diverse arrangements and coordination environments. Similar multiple phases have been reported in Na-intercalated FeSe superconductors [41]. When the thin flake is tuned to an insulator, the 11 phase and 122-I phase completely disappear and only 122 -II phase exists as shown in Fig. 3. When the gate voltage is swept back to $-2 \mathrm{~V}$, the $122-\mathrm{I}$ and $-\mathrm{II}$ phases completely disappear, and the FeSe phase recovers. This result indicates that the newly formed 122 structural phases can only exist under the electric field, and also implies that the process of intercalating $\mathrm{Li}^{+}$into the FeSe controlled by electric field is reversible. We note that the two new Li-intercalated FeSe phases have not been reported before, which are induced and stabilized by the SIC-FET device. Since the superconductivity in bulk FeSe-derived materials is primarily determined by carrier concentration doped into FeSe layers, the emerging new 122 structures may not play a primary role in the novel electronic properties of FeSe layers. However, the superconductivity and other properties, and especially the insulating behavior of these new 122 phases is very intriguing for further investigation.

To acquire more details about the electron doping and structural modification, in-situ X-ray photoelectron spectroscopy (XPS) and X-ray absorption near edge spectra (XANES) were performed on the SIC-FET devices to study FeSe thin flakes with different lithium contents. The XPS result for Fe $2 p$ is shown in Fig. S5. The spectrum taken on FeSe single crystals agrees well with that of the as-grown FeSe film [42]. It consists of two features at $706.6 \mathrm{eV}$ and $707.8 \mathrm{eV}$. We use the center of the weight of the $\mathrm{Fe} 2 p$ peaks to qualitatively describe the change of Fe chemical valence. The center point decreases from $707.1 \mathrm{eV}$ (no gating), $707.0 \mathrm{eV}$ (optimal superconductivity) and eventually to $706.6 \mathrm{eV}$ (insulating phase). Clearly, the Fe valence state decreases with the increase of $\mathrm{Li}^{+}$ions intercalation. Due to the lack of reference data and surface sensitive nature of XPS, it is difficult for us to determine the Fe valance accurately at this moment. In addition, the XANES Fe $K$-edge spectra are shown in Fig. S6. This main absorption feature is due to the transition from $\mathrm{Fe} 1 s$ to the $4 p$ state. The absorption edge of the insulating phase FeSe thin flake shifts $2.5 \mathrm{eV}$ towards lower energy relative to that of pure FeSe, corresponding to a change of the $\mathrm{Fe}$ valence state from +2 in the pure FeSe to $1.23 \pm 0.15$ in the insulating phase. Furthermore, the slight parallel shift of absorption edge at the optimal superconducting state gives additional evidence for the random distribution of $\mathrm{Li}$ over $\mathrm{Fe}$ since a new local ordering may give rise to the deviations in the absorption spectra. On the other hand, the distinct shift of the absorption edge in the insulating phase from that of pure FeSe sample indicates a new ordering or structural change due to Li intercalation. Such a change is also responsible for the variation of the pronounced preedge feature at $7111 \mathrm{eV}$, which arises from the quadruple transition from $\mathrm{Fe} 1 s$ to $\mathrm{Fe} 3 d$. These findings support the formation of a new 122 structure phase in the insulating state. The decrease of $\mathrm{Fe}$ valence state observed in both of XPS and XANES suggests that $\mathrm{Li}^{+}$intercalation controlled by electric field is an effective method to introduce electron carriers into FeSe layers. The XANES results indicate that the $\mathrm{Li}$ is randomly distributed in 122-I phase, while is ordered in 122-II phase. Further investigation is required to determine $\mathrm{Li}$ positions and to reveal their microstructures in the two phases.

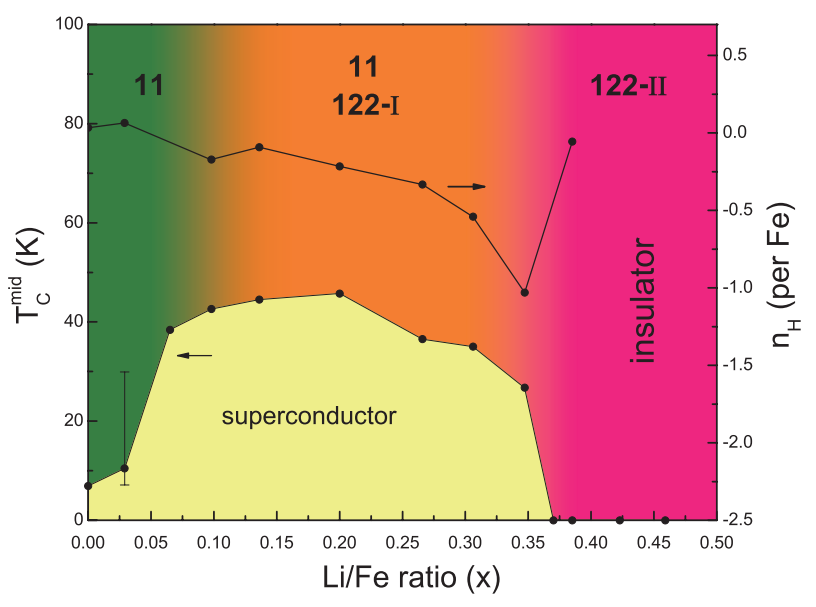

FIG. 4: (color online). The phase diagram of Li-intercalated FeSe thin flake as a function of $\mathrm{Li} / \mathrm{Fe}$ ratio. The $T_{c}$ shows a dome-like behavior with increasing $\mathrm{Li} / \mathrm{Fe}$ ratio. A phase transition from superconductor to insulator happens around $\mathrm{Li} / \mathrm{Fe}$ ratio of 0.37 . A series of structural phase transitions from $\mathrm{FeSe} 11$ phase to $\mathrm{Li}_{y} \mathrm{Fe}_{2} \mathrm{Se}_{2}$-I phase, then to $\mathrm{Li}_{y} \mathrm{Fe}_{2} \mathrm{Se}_{2}$ II phase takes place. The Hall number $\mathrm{n}_{H}$ is plotted to show the variation of carrier density.

The phase diagram of superconductivity and phase variations of $\mathrm{Li}^{+}$intercalated FeSe thin flakes are plotted as a function of $\mathrm{Li} / \mathrm{Fe}$ ratio in Figure 4. The method of determining the amount of intercalated $\mathrm{Li}^{+}$content is described in the Supplemental Material. Based on the experimental results, the SIC-FET not only tunes the carrier concentration of the samples, but also modulates the crystal structure of materials. With increasing the amount of intercalated $\mathrm{Li}^{+}$, the $T_{c}$ shows a dome-like be- 
havior. The optimal superconductivity with mid-point critical temperature $T_{c}^{\text {mid }}=45.8 \mathrm{~K}$ has been achieved when the $\mathrm{Li} / \mathrm{Fe}$ ratio reaches 0.2 . As the ratio is up to about 0.37 , the superconductivity disappears completely, and then the sample becomes an insulator. A similar evolution from superconductor to insulator has been also observed in $(\mathrm{Li}, \mathrm{Fe}) \mathrm{OHFeSe}$ thin flake by electric field controlled lithium doping [43]. Most intriguingly, an abrupt jump of the $T_{c}$ from $\sim 10 \mathrm{~K}$ to $\sim 40 \mathrm{~K}$ is observed when the $\mathrm{Li} / \mathrm{Fe}$ ratio is around 0.07. Meanwhile, the Hall number $\mathrm{n}_{H}$ shows a corresponding sudden sign reversal. The evolution of Hall signal from hole-type to electron-type upon the increase of electron concentration induced by $\mathrm{Li}^{+}$intercalation can serve as an indicative of Lifshitz transition [4]. Similar transition has been observed in FeSe thin flakes with EDL-FET device [39]. However, for a comparison, the effective carrier concentration introduced by $\mathrm{Li}^{+}$intercalation can further increase and go beyond the upper limit of EDL-FET devices. As shown in Fig. 4, the carrier concentration suddenly decreases when the sample is tuned to an insulator, which could be attributed to some charge localization effect. In the whole phase diagram, the system experiences a series of phase transitions. In the beginning of $\mathrm{Li}^{+}$intercalation into FeSe thin flakes, the system keeps the same structure as FeSe, and a sudden enhancement of $T_{c}$ occurs. With further increasing $\mathrm{Li}^{+}$intercalation, the 122 -I phase emerges and coexists with the FeSe phase, then 122-II phase shows up at higher doping level. When the system is tuned to an insulator, FeSe phase and 122-I phase completely disappear, and only 122 -II phase remains. We note that the coexistence of the 11, 122-I and -II phases is not intrinsic due to the inhomogeneity of the $\mathrm{Li}^{+}$distribution. Such inhomogeneous distribution of $\mathrm{Li}^{+}$in thin flakes strongly depends on the thickness of thin flakes, which was confirmed by the XRD patterns shown in Fig. 3 and Fig.S5 for thin flakes with different thickness of 40 $\mathrm{nm}$ and $150 \mathrm{~nm}$, respectively. It should be pointed out that the data shown in Fig. 2 were obtained on the FeSe thin flakes of $18 \mathrm{~nm}$, which should be much more homogeneous than that observed in Fig. 3. It is possible that no coexistence of the 11, 122-I and -II phases takes place in the devices with the flakes of $18 \mathrm{~nm}$. We have tried to perform XRD measurements on the device with the $18 \mathrm{~nm}$ thin flakes by XRD, but the sample is too thin to detect signal. We thus selected a $40 \mathrm{~nm}$ FeSe thin flake for XRD characterization, which could inevitably involve inhomogeneity.

In summary, using solid lithium ion conductor as the gate dielectric, we developed a novel FET device (SICFET), which can effectively introduce electron carriers into layered materials. Compared to the MIS-FET and the EDL-FET devices, the SIC-FET device shows stronger capability of tuning carrier concentration, so that it can help to map out the phase diagram of FeSe over a wide range for the first time. Owing to the small ionic radius of $\mathrm{Li}^{+}$, the SIC-FET device can tune the carrier concentration of entire materials with thickness of ten nanometers. Besides, the SIC-FET device can be used to search for new materials or novel superconductors with metastable structures. In particular, the configuration of SIC-FET devices is suitable for many other surface-sensitive experimental examinations. In our studies, two novel Li-intercalated FeSe phases with $\mathrm{ThCr}_{2} \mathrm{Si}_{2}$-type structure, which cannot be synthesized by conventional methods, were obtained under the electric field. The investigation on the insulating $\mathrm{Li}_{y} \mathrm{Fe}_{2} \mathrm{Se}_{2}$-II phase could be helpful for the understanding of the superconducting mechanism for FeSe-derived superconductors. Our findings demonstrate the potential of the SIC-FET device to control superconductivity and to modulate the crystal structure, and open a new way to search for novel electronic or structural phases. Because of its significant advantage, the SIC-FET can serve in the modern electronic technology to some degree.

This work is supported by the National Natural Science Foundation of China (Grants No. 11190021, No. 11227902, No. 11534010 and No. 91422303), the Strategic Priority Research Program (B) of the Chinese Academy of Sciences (Grant No. XDB04040100), the National Key R\&D Program of the MOST of China (Grant No. 2016YFA0300201), and the Hefei Science Center CAS (2016HSC-IU001).

* Electronic address: zhyb@fudan.edu.cn

† Electronic address: chenxh@ustc.edu.cn

[1] J. G. Bednoz and K. A. Müller. Z. Phys. B 64, 189-193 (1986).

[2] Y. Kamihara et al., J. Am. Chem. Soc. 130, 3296-3297 (2008).

[3] X. H. Chen et al., Nature 453,761-762 (2008).

[4] R. E. Glover et al., Phys. Rev. Lett. 5, 248-250 (1960).

[5] C. H. Ahn et al., Science 284, 1152-1155 (1999).

[6] D. Chiba et al., Science 301, 943 (2003).

[7] C. H. Ahn et al., Nature 424, 1015-1018 (2003).

[8] K. S. Novoselov et al., Science 306, 666 (2004).

[9] A. D. Caviglia et al., Nature 456, 624-627 (2008).

[10] K. Ueno et al., Nat. Mater. 7, 855 (2008).

[11] K. Ueno et al., Nat. Nanotechnol. 6, 408 (2011).

[12] J. T. Ye et al., Nat. Mater. 9, 125-128 (2010).

[13] A. T. Bollinger et al., Nature 472, 458-460 (2011).

[14] J. T. Ye et al., Science 338, 1193-1196 (2012).

[15] Y. J. Yu et al., Nat. Nanotechnol. 10, 270 (2015).

[16] Y. Saito et al., Science 350, 409-413 (2015).

[17] J. M. Lu et al., Science 350, 1353-1357 (2015).

[18] L. J. Li et al., Nature 529, 185-190 (2016).

[19] B. Radisavljevic et al., Nat. Nanotechnol. 6, 147-150 (2011).

[20] L. K. Li et al., Nat. Nanotechnol. 9, 372 (2014).

[21] C. H. Ahn et al., Rev. Mod. Phys. 78, 1185-1212 (2006).

[22] K. Ueno et al., Journal of the Physical Society of Japan 83, 032001 (2014).

[23] H. T. Yuan et al., Adv. Funct. Mater. 19, 1046-1053 
(2009).

[24] J. Jeong et al., Science 339, 1402-1405 (2013).

[25] T, D. Schladt et al., ACS Nano 7, 8074-8081 (2013).

[26] F. C. Hsu et al., Proc. Natl. Acad. Sci. USA 105, 1426214264 (2008).

[27] J. G. Guo et al., Phys. Rev. B 82, 180520(R) (2010).

[28] A. F. Wang et al., Phys.Rev.B 83, 060512(R) (2011).

[29] M. Burrard-Lucas et al., Nat. Mater. 12, 15-19 (2013).

[30] X. F. Lu, et al., Nat. Mater. 14, 325-329 (2015).

[31] Q. Y. Wang et al., Chin. Phys. Lett. 29, 037402 (2012).

[32] D. F. Liu et al., Nat. Commun. 3, 931 (2012).

[33] S. L. He et al., Nat. Mater. 12, 605-610 (2013).

[34] S. Y. Tan et al., Nat. Mater. 12, 634-640 (2013).
[35] R. Peng et al., Nat. Commun. 5, 5044 (2014).

[36] G-F. Ge et al., Nat. Mater. 14, 285-289 (2015).

[37] L. Zhao et al., Nat. Commun. 7, 10608 (2016).

[38] X. H. Niu et al., Phys. Rev. B 92, 060504(R) (2015).

[39] B. Lei et al., Phys. Rev. Lett. 116, 077002 (2016).

[40] K. Hanzawa et al., Proc. Natl. Acad. Sci. 113, 3986-3990 (2016).

[41] J. Guo et al., Nat. Commun. 5, 4756 (2014)

[42] X. D. Qi et al., Journal of Alloys and Compounds 509, $6350 ? 353$ (2011).

[43] B. Lei et al., Phys. Rev. B 93, 060501(R) (2016).

[44] I. M. Lifshitz, Sov. Phys. JETP 11, 1130 (1960). 